\title{
Dynamics of forced biopolymer translocation
}

\author{
V.V. LEHTOLA, R.P. LINNA and K. KASKI \\ Department of Biomedical Engineering and Computational Science, \\ Helsinki University of Technology, \\ P.O. Box 9203, FIN-02015 TKK, Finland
}

\begin{abstract}
PACS $87.15 .-\mathrm{v}-$ Biomolecules: structure and physical properties
PACS 82.35 . Lr - Physical properties of polymers

PACS $87.15 . \mathrm{A}--$ Theory, modeling, and computer simulation
\end{abstract}

\begin{abstract}
We present results from our simulations of biopolymer translocation in a solvent which explain the main experimental findings. The forced translocation can be described by simple force balance arguments for the relevant range of pore potentials in experiments and biological systems. Scaling of translocation time with polymer length varies with pore force and friction. Hydrodynamics affects this scaling and significantly reduces translocation times.
\end{abstract}

The transport of biopolymers through a nano-scale pore in a membrane is a ubiquitous process in biology. For example, in protein import into mitochondria, chloroplasts, and peroxisomes the translocation occurs with the aid of a membrane , potential [1]. Experimental work on forced (or biased) translocation is largely motivated by finding methods for reading the DNA and RNA sequences. These nanopores are typically either fabricated solid-state [2,3] or $\alpha$-hemolysin $(\alpha-\mathrm{HL})$ pores ' in lipid bi-layer membranes $[4,5]$. Foundation for the theoretical work was laid in the classic treatment by Sung and Park [6], which was based on the assumption that the polymer segments on the two sides of the membrane reside close to separate thermal equilibria. However, the validity of this approach was questioned already in $[7,8]$, where the authors 'noted that the pore force regime in which the polymer's relaxation time towards equilibrium is smaller than the characteristic translocation time is marginal and that the approach would be invalid even in the unforced translocation for sufficiently long polymers. Theoretical work, inconsistent with experiments, has since evolved in different directions.

The role of computer simulations has been largely to support the theoretical work which neglects hydrodynamics. Hence, results from simulations where hydrodynamic interactions are included are few and, due to their being computationally demanding, often fairly qualitative $[9,10]$. In addition, the generally used Monte Carlo method gives unphysical behaviour for larger pore force values relevant for experiments and biological systems $[2,4,5]$, as we have shown [11]. Very recently multiscale simulations on biopolymer translocation in a solvent were reported to give results in accordance with experiments [12,13].
Our motivation for the present study is two-fold. First, by using realistic dynamics we want to find explanation for the dynamics of the experimentally observed translocation processes. Secondly, we want to determine the effect of hydrodynamics on forced polymer translocation, previously studied only in the unforced case $[9,10]$. We use a hybrid multi-scale method, where the polymer follows detailed molecular dynamics and the coarse-grained solvent stochastic rotation dynamics (SRD). The solvent is divided into cells, within which fictitious solvent particles perform simplified dynamics where collisions among them and with the polymer beads are taken effectively into account by performing random rotations of the random part of their velocities, $v_{i}\left(t+\Delta t_{S R D}\right)=\mathbf{R}\left[v_{i}(t)-v_{c m}(t)\right]+v_{c m}(t)$, where $v_{i}$ are the particle velocities inside a cell, $\Delta t_{S R D}$ is the time step for solvent dynamics, $\mathbf{R}$ is the rotation matrix, and $v_{c m}$ is the centre-of-mass velocity of the particles within the cell. Hydrodynamic modes are supported over the cells. Optionally, they can be switched off by not adding back $v_{c m}$ after the random rotation, which is particularly feasible for pinning down the effect of hydrodynamics. The abovedescribed collision step is followed by the free-streaming step $r_{i}\left(t+\Delta t_{S R D}\right)=r_{i}(t)+v_{i}(t) \Delta t_{S R D}$. Thermostating is done by rescaling all solvent particle velocities so that equipartition theorem is fulfilled at all times. More detailed descriptions of the method can be found e.g. in [14-16].

In this paper we study the forced translocation where the two sides separated by walls are not hydrodynamically coupled. To achieve this we use a non-aqueous pore, i.e. there are no solvent particles inside the pore. This corresponds closely to the experiments we aim to model and also addresses the theoreti- 
cal predictions, where the two subspaces separated by the wall are taken to be uncoupled. In addition, technically speaking the coarse-grained solvent dynamics does not allow for overly confined spaces, but implementing solvent dynamics in dimensions smaller than the SRD cell dimension would require detailed molecular dynamics for the solvent. The linear SRD cell dimension in our model is $\Delta x \equiv 1.0 \equiv b$, where $b$ is the polymer bond length.

The standard bead-spring chain is used as a coarse-grained polymer model [11,17]. Adjacent monomers are connected with anharmonic springs, described by the finitely extensible nonlinear elastic (FENE) potential,

$$
U_{F E N E}=-\frac{K}{2} R^{2} \ln \left(1-\frac{r^{2}}{R^{2}}\right) .
$$

Here $r$ is the length of an effective bond, $R=1.5$ the maximum bond length. The Lennard-Jones (LJ) potential

$$
\begin{aligned}
U_{L J} & =4 \epsilon\left[\left(\frac{\sigma}{r}\right)^{12}-\left(\frac{\sigma}{r}\right)^{6}\right], r \leq 2^{-1 / 6} \sigma \\
U_{L J} & =0, r>2^{-1 / 6} \sigma,
\end{aligned}
$$

is used between all beads. The parameter values were chosen to be $\epsilon=1.2, \sigma=1.0$, and $K=60 / \sigma^{2}$. The used LJ potential with no attractive part mimics good solvent condition for the polymer.

We do not include a harmonic bending potential, which would change the above-described freely-jointed chain (FJC) to the worm-like-chain (WLC). Elastically, the FJC and WLC were seen to differ only marginally and their hydrodynamic characteristics were found identical in the present model [17]. The swelling exponents measured for the FJC and WLC in the present model were identical, which means that since packaging of polymers is not an issue in the present geometry, as e.g. in capsids [18], the bending potential does not constitute an important factor to the translocation process.

The model geometry contains a slif formed by two walls perpendicular to the $x$-direction. Periodic boundary conditions are applied in $y$ - and $z$-directions. A third wall, three polymer segments, $b$, thick and impermeable to the solvent, is placed in the middle of the system, perpendicular to the $z$ direction. No slip boundary conditions are applied between the three walls and the solvent. A circular nanopore of diameter $1.2 b$ is placed in the centre of the middle wall. The force $f$ acting on the beads inside the nanopore is constant and local for the pore, which models well the experimental setups and biological systems, where solvents are good ionic conductors eliminating any potential gradients outside the pore. The polymer beads inside the pore are not coupled with hydrodynamic modes or with the heat bath and in the directions perpendicular to the cylindrical pore walls experience a damped harmonic potential $U_{h}$, described by $-\nabla U_{h}=F_{h}=-k r_{x, y}-c v_{x, y}$, where $k=1000$, $c=10, r_{x, y}$ is the polymer bead position with respect to the centre axis of the cylindrical pore, and $v_{x, y}$ is the velocity component perpendicular to the pore walls. Thus $U_{h}$ centres the

\footnotetext{
${ }^{1}$ The slit mimics experimental setups including confining walls. The slit is large enough not to affect dynamics of the translocating polymer.
}

polymer along the $z$-directional axis of the pore. The potential is chosen large, so no hairpin configuration can enter the pore as its width is effectively small. Hence, the polymer segment inside the pore remains rather straight. In the $z$ direction, the polymer beads experience either zero or finite friction in the pore. In the zero friction case the polymer beads inside the pore are moved by a constant force included in the molecular dynamics and the momentum is conserved in the SRD step performed every 50th MD step. In order to investigate the effect of pore friction the momentum was optionally destroyed in the SRD step in a manner similar to switching off hydrodynamic modes in the solvent. This amounts to the polymer beads experiencing finite friction inside the pore.

Diffusion of the chains and single particles has been verified to satisfy equipartition theorem in equilibrium. The swelling exponent value of a self-avoiding-chain, $\nu=0.6 \pm 0.05$, was measured and the fluctuations of the radius of gyration the polymer configurations in directions aligned with and perpendicular to the side walls were measured and found equal [17]. Thus the side walls do not affect the dynamics of polymers. Translocation simulations were started from initial configurations that were checked to be in equilibrium with respect to the radius of gyration, $R_{g}$. The measured $R_{g}$ 's were clearly smaller than the channel dimensions, which excludes effects due to polymer confinement. Also, finite (simulation box) size effects were verified not to affect the dynamics by measuring the relaxation time $\tau_{r} \sim\left\langle R_{g}(t) R_{g}(0)\right\rangle$ for polymers of length $N=200$ and various volumes (box sizes). The relaxation times are of the same order than the largest translocation times presented in Fig. 11b). The simulation box sizes are $[25,32,32]$ for $N \leq 50$, $[32,32,32]$ for $N \leq 100,[40,32,32]$ for $N \leq 200,[40,40,40]$ for $N \leq 400$, and $[60,60,60]$ for $N=800$. The measured radii of gyration are $4.91 \pm 0.01$ for $N=50,7.19 \pm 0.01$ for $N=100$, and $10.93 \pm 0.01$ for $N=200$.

In order to characterise the native translocation process, we use pore force values that are sufficient to induce translocation, hence obviating any additional constraints for preventing the polymer from sliding back to the cis side (see a snapshot of a translocating polymer in Fig. 2 c c)). As has been noted, additional constraints can potentially change the observed scaling of the translocation time $\tau$ with polymer length $N$ [19]. We compare our simulated results to experiments $[2,4,5]$ and also to those obtained analytically from the Brownian translocation framework $[6,20]$ and the numerical results supporting anomalous diffusion $[8,10,21]$. The assumption of the whole or parts (called folds) of the polymer close to the pore being in equilibrium is crucial to the application of the aforementioned frameworks. We show that these assumptions are invalid in the experimental pore force range and that hydrodynamics has a profound effect on the forced translocation. This, in turn, we show to be a highly non-equilibrium process governed by a simple force balance closely related to the one presented by Storm et al., albeit with important modifications.

We present the results from our simulations in reduced, dimensionless units. The unit of length is defined as the polymer bond length, $b$, which corresponds roughly to the Kuhn length of the translocating polymer in SI-units, $\tilde{b}$. For our freely- 

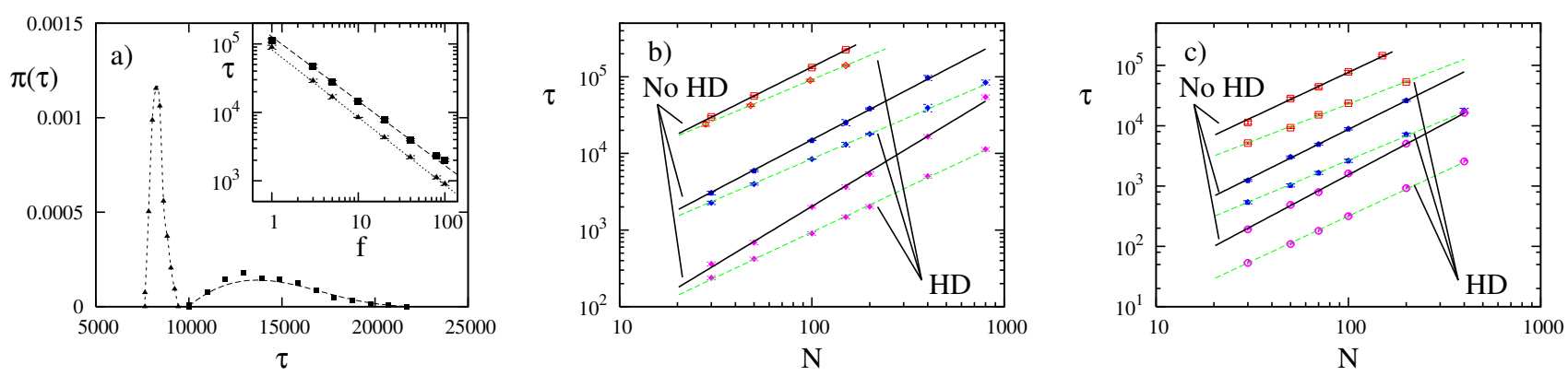

Fig. 1: (Colour online) a) The distribution of translocation times $\tau$ for chains of length $N=100$ and a constant pore force $f=10$, with hydrodynamics $(\boldsymbol{\Lambda})$ (averaged over 300 runs) and without hydrodynamics (ם) (averaged over 400 runs). Inset: Average translocation time $\tau$ as a function of the driving force $f$. The scaling $\tau \sim f^{\alpha}$ is obtained with (⿴囗十) $\alpha=-0.940 \pm 0.013$ for $f \geq 3$, and $(\mathbf{\Lambda}) \alpha=-0.994 \pm 0.008$ for $f \geq 1$ without and with hydrodynamics, respectively. The chain length is $N=100$, and the pore is frictional. b) \& c) Average translocation time $\tau$ as a function of the chain length $N$ with b) frictional pore and c) frictionless pore. Results are displayed both with and without hydrodynamics (HD). b) Shown are $\tau \sim N^{\beta}$ for forces $f=1,10,100$ (from top to bottom). For forces $f=1,3,10,40,100$, $\beta=1.25 \pm 0.02,1.26 \pm 0.02,1.31 \pm 0.02,1.43 \pm 0.03,1.52 \pm 0.03$ without $\mathrm{HD}$ and $\beta=1.05 \pm 0.02,1.04 \pm 0.01,1.07 \pm 0.01,1.13 \pm 0.01$, $1.18 \pm 0.02$ with HD. c) Shown are $\tau \sim N^{\beta}$ for forces $f=1,10$ (from top to bottom). For forces $f=1,3,10,100, \beta=1.50 \pm 0.04$, $1.50 \pm 0.03,1.58 \pm 0.03,1.70 \pm 0.03$ without $\mathrm{HD}$, and $\beta=1.23 \pm 0.03,1.26 \pm 0.02,1.33 \pm 0.02,1.48 \pm 0.02$ with HD.

jointed chain (FJC) the Kuhn length can be taken as $\tilde{b}=2 \lambda_{p}$, where $\lambda_{p}$ is the persistence length, roughly $40 \AA$ for a singlestranded (ss) and $500 \AA$ for a double-stranded (ds) DNA [22]. In the simulations the force is exerted on three beads residing simultaneously inside the pore. The pore force per bead in SI-units, $\tilde{f}$, is obtained from the dimensionless force per bead, $f$, as $\tilde{f} \equiv f k_{B} T / \tilde{b}$. The simulations were performed at $k_{B} T=1$, which we take to correspond to $\tilde{T}=300 \mathrm{~K}$. Hence, the dimensionless force $f=1$ corresponds to the total pore force $\tilde{f}_{t o t}=3 \tilde{f}$, giving $\tilde{f}_{t o t} \approx 0.12 \mathrm{pN}$ for ds DNA and $\tilde{f}_{\text {tot }} \approx 1.6 \mathrm{pN}$ for ssDNA. A typical experimentally used potential driving a polymer through the pore for both the ssDNA in the $\alpha-\mathrm{HL}$ and dsDNA in the solid state pore is $\sim 120 \mathrm{mV}$, which would give a pore force of $\sim 50 \mathrm{pN}$ for ssDNA and $\sim 110 \mathrm{pN}$ for dsDNA. When charge reduction due to Manning condensation is taken into account, the effective force for dsDNA in the solid-state pore was evaluated to be in the range $20-50 \mathrm{pN}[2,23]$. For ssDNA in an $\alpha$-HL pore the charge reduction was evaluated to be even more drastic due to confinement in the pore in addition to the normal charge reduction, giving $\tilde{f} \sim 5 \mathrm{pN}[4,24]$. This would suggest that also the estimated force for dsDNA could be smaller. The translocation of a polymer across a pore in a biological membrane involves in addition friction and e.g. interaction of the polymer with the pore proteins $[25,26]$, which without detailed information on those interactions makes exact mapping of the pore force values used in the simulation to those in experiments impossible. The primary control parameter is the total pore force, $\tilde{f}_{\text {tot }}=M \tilde{f}$, where $M$ is the number of points on the polymer contour on which the pore force, $\tilde{f}$, is exerted. On dsDNA these points can be taken to reside at intervals determined by the nucleotide spacing, which is $3.4 \AA$ for dsDNA and $\approx 4 \AA$ for ssDNA. The pore force per bead in the experiments may be estimated as $\tilde{f}=z q^{*} V / L$, where the pore potential $V=120 \mathrm{mV}$ and the number of elementary charges $e$ per nucleotide is $z=2$ for dsDNA and $z=1$ for ssDNA. The effective charge $q^{*}$ is taken as $e$ for dsDNA [2] and 0.1e for ssDNA due to charge reduction [24]. This gives $\tilde{f} \approx 1.92 \mathrm{pN}$ for dsDNA and $\tilde{f} \approx 0.37 \mathrm{pN}$ for ssDNA. Since the length of the solid state pore is $20 \mathrm{~nm}$, $M \approx 59, \tilde{f}_{t o t} \gtrsim 113 \mathrm{pN}$ for dsDNA, but could be considerably smaller as pointed out above [2]. The length of the $\alpha$-HL pore is $52 \AA$, so $M \approx 13$, giving $\tilde{f}_{t o t} \approx 5$ pN for ssDNA [4]. So in summary, in spite of the intricacies involved in estimating the true force exerted on the polymer inside the pore, the experimental force magnitudes are included in the pore force range $f \in[1,100]$ used in our simulations and, what is more important with respect to the observed of out-of-equilibrium effects pertinent to the forced translocation, the minimum pore force used in our simulations is well under the minimum pore force magnitudes used in experiments.

For the frictional pore we obtained the translocation probability of $P_{t r}=0.12 \pm 0.05$ for $f=0.25$ that can be taken as a crude estimate for the minimum force required for generic forced translocation. $P_{t r}$ reached unity at $f \approx 1$. For a frictionless pore $P_{t r} \sim 0.5$ with $f \simeq 1$, and $P_{t r}=1$ at $f \approx 2$. Experiments on protein translocation across inner mitochondrial membrane, where the pore is highly frictional, showed saturation of $P_{t r}$ with the pore potential $80 \mathrm{mV}$ [25]. The potential in related experiments varied from 150 to $240 \mathrm{mV}$ [27,28]. Hence, in terms of generic translocation, the pore force used in our simulations is in a range relevant for experiments. The simulated pores were non-aqueous. Having solvent inside the pore may change the minimum pore force value inducing translocation. This will be investigated in a future publication. Experimentally, an average velocity of $\langle v\rangle \sim 1 \mathrm{~cm} / \mathrm{s}$ was measured for a dsDNA translocating across a solid-state pore with a typical potential of $\sim 120 \mathrm{mV}$ (corresponding to a pore force of $20-50 \mathrm{pN}$ ) [2,3]. We obtained an average velocity of $v=0.004$ for $f=1$, so the dimensionless simulation time unit corresponds roughly to $\tilde{t} \sim 0.1 \mu \mathrm{s}$. In our translocation simulations SRD step is performed every 50th MD step, so the solvent and polymer time steps are $\Delta t_{S R D}=0.1 \Delta t$ and $\Delta t_{M D}=$ 
$0.002 \Delta t$, respectively. We obtain $\eta \approx 15.776$ for the viscosity of our model [29]. For a polymer of length $N=100$ we measured radius of gyration of $R_{g} \approx 7.198$. Hence we calculate for the Zimm relaxation time [30], i.e. the time it takes for the entire polymer to relax to an entropically and sterically favourable configuration, $t_{z}=0.398 \eta R_{g}^{3} / k_{B} T \approx 2.4 \cdot 10^{3}$. Estimating the friction coefficient for our model [29] to be $\zeta \approx 25$, we obtain for the corresponding (Rouse) relaxation time without hydrodynamics [30] $t_{r}=\zeta(N b)^{2} / 3 \pi^{2} k_{B} T \approx 8.5 \cdot 10^{3}$. So, in our model the estimated ratio of relaxation times with and without hydrodynamics of a polymer of length $N=100$ is $R=t_{z} / t_{r} \approx 3.5$. We measured $R \approx 1.6$. The singleparticle Reynold's number for this velocity in our model [29] is $R e=0.005$, which is in the relevant regime for physiological solvents.

First we determine the translocation time, $\tau$, as a function of pore force, $f$. For the scaling exponents $\alpha$ defined as $\tau \sim f^{\alpha}$ we obtain $\alpha=-0.940 \pm 0.013$ for $f \in[3,100]$ and $\alpha=-0.994 \pm 0.008$ for $f \in[1,100]$ without and with hydrodynamics, respectively, see the inset of Fig. 1 a). Hence, essentially $\tau \sim f^{-1}$ was obtained, as was to be expected for force values large compared with thermal fluctuations.

The distribution of translocation times, $\pi(\tau)$, for polymers of length $N=100$ is shown in the main part of Fig. 1 a). Due to the larger polymer velocities in forced translocation the effect of hydrodynamics on forced translocation is much more pronounced than what has been seen with unforced translocation $[9,10]$. We obtain a reduction in translocation times due to hydrodynamics, which was also seen by Fyta et al [13,31]. In addition, hydrodynamics not only significantly speeds up forced translocation but also reduces the variance of measured translocation times, which is induced by the long range correlations due to hydrodynamics, mediating the effect of the pore force along the polymer contour.

The measured translocation times scale with polymer length, $\tau \propto N^{\beta}$, both with and without hydrodynamics, see Figs. 11 b) and c). It is noteworthy, however, that there exists no single scaling, but $\beta$ varies with pore force, $f$. In our simulations, $\beta$ starts from unity and increases with $f$. At constant $f$, smaller $\beta$ was obtained for the frictional pore. To distinguish between the change of $\beta$ due to increasing translocation velocity, $v$, and due to frictional term, when $f$ was increased, scaling of $\tau$ with $N$ for a pore with no friction was measured and it was found that the change of $\beta$ was still significant, see Fig. 1. The experimentally obtained $\beta \approx 1.27$ for a solid state pore [2] would be obtained in our model with a pore force $f \gtrsim 3$. Hence, it can be concluded that the change of $\beta$ with $f$ arises not only from the change in the frictional contribution to the translocation dynamics, but also because of dynamic changes due to the change in $v$, which is a clear indication of out-of-equilibrium effects. In comparison, Fyta et al. [13] obtained $\beta=1.28 \pm 0.01$, and $\beta=1.36 \pm 0.03$ with and without hydrodynamics, respectively, for the pore force $f=1$, which closely corresponds to $f=1$ in our simulations as the pore length in [13] is approximately $3 b$. A pore of very low friction was used in these lattice Boltzmann (LB) simulations. Accordingly, the obtained scaling exponent is in fair agreement with ones we have obtained for the frictionless pore with hydrodynamics. Also the increase of $\beta$ when hydrodynamics is switched off qualitatively agrees with our results. Fyta et al. report the scaling exponent only for a single pore force magnitude. The verification of possibly nonuniversal scaling exponents varying with the pore force using the lattice Boltzmann method would be most valuable.

Using Langevin dynamics (where hydrodynamics is excluded) we checked that linear scaling $(\beta=1)$ can be achieved for any constant pore force with large enough friction. Linear scaling has been seen with $\alpha$-HL pores, whose diameter is smaller and friction larger than those of solid state pores. The important notion is that $\beta$ varies with pore friction even at moderate friction values, regardless of hydrodynamic interactions.

Most of our results are for a frictional pore, which is the more realistic. We use pore force magnitudes $f \geq 1$ at which translocation takes place with very high probability $\left(P_{t r} \approx 1\right)$ and hence we do not address unforced translocation. Without hydrodynamics, the translocation with a frictionless pore approaches the scaling $\tau \sim N^{1+\nu} \approx N^{1.6}$ as $f$ increases. $\beta=1+\nu$ is the scaling exponent predicted by the Brownian translocation framework, independent of $f$. $\beta$ can be increased further by applying an unrealistically large pore force. This increase is due to crowding on the trans side, discussed further below.

When hydrodynamics is allowed, the polymer segments are moved from their initial equilibrium positions already before actually being pulled by the tightening polymer contour. This is seen in Fig. 2 a), where the squared distance, $R_{p e}^{2}(n)$, of the polymer bead, labelled $n$, measured from the pore on the cis side as a function of the number of translocated beads, $s$, is shown. In the absence of hydrodynamics, the segments towards the free end are seen to remain immobile until they are pulled towards the pore, whereas due to hydrodynamic interactions the distance of the labelled bead $n$ from the pore is seen to start decreasing right from the beginning of the translocation. Hence, the initial configuration shows less in the translocation, when hydrodynamics is included. Instead, the configuration on the cis side continually changes towards increasingly extended one. Regarding only the dynamics on the cis side, for an initially completely extended polymer asymptotically $\beta \rightarrow 1$ as $f$ is increased, which explains the reduction of $\beta$ at constant $f$ when hydrodynamics is applied. Evidently, mechanisms like squeezing of equilibrated folds through the pore, suggested in [21,32], contradict with this mode of motion, where alignment of segments in the vicinity of the pore is to be expected and, indeed, seen in the snapshots, see Fig. 2 c).

In order to identify the underlying mechanism in the forced translocation, we extract from the measured distances of the labelled polymer beads from the pore, $R_{p e}$ the number of mobile beads, $s_{m}$. We define a labelled bead as mobile if this measured distance, averaged over several runs, changes appreciably. The number of mobile beads, $s_{m}$, as a function of translocated beads $s$ is read off from the inflection points in Fig. 2] a) depicting the measured $R_{p e}^{2}(s / N)$ in the case of no hydrodynamic interactions. In Fig. 2 b) $s_{m}$ is plotted as a function of translocated beads $s$ when hydrodynamics is not included. Linear dependence $s_{m}=k s$ is obtained. Up to lengths of 

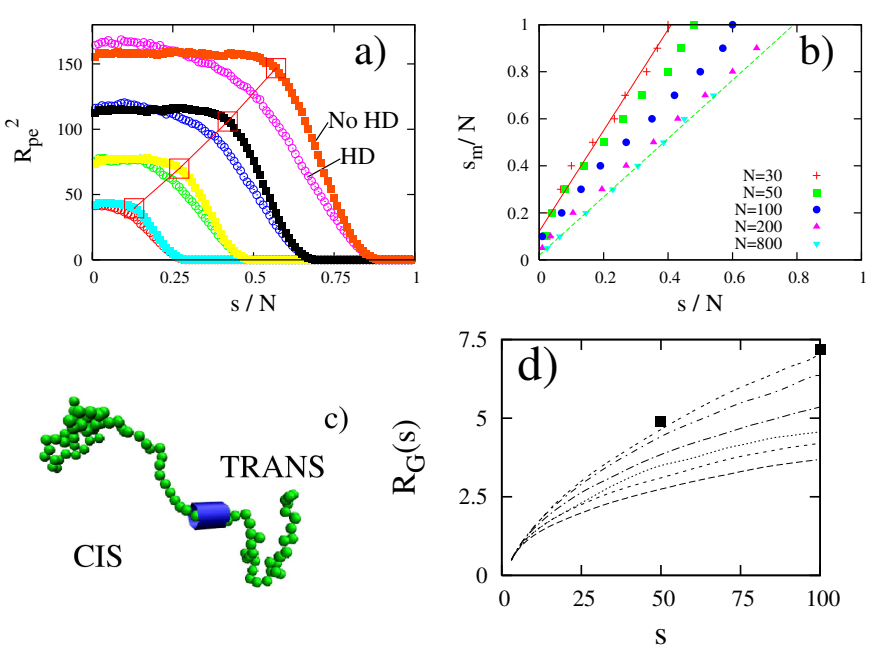

Fig. 2: (Colour online) a) Averaged squared distances of beads numbered $30,50,70$, and 90 from the pore as a function of the number of translocated beads $s$ for polymers of length $N=100$ with and without hydrodynamics. b) The number of mobile beads, $s_{m}$, (see text) vs number of translocated beads, $s$, both normalised to the polymer length, $N$, in the case of no hydrodynamics. $f=10$ in a) and b). c) $3 \mathrm{D}$ snapshot of a translocating polymer of length $N=100$ at $s=35$. Here the pore is frictionless and $f=2$. For clarity reasons, walls are not shown. d) The radius of gyration $R_{g}$ on the trans side as a function of the number of translocated segments $s$. Chain length $N=100$. The applied pore forces for the curves from top to bottom are 1,3,10,20,40, and 100. Measured equilibrium $R_{g}$ 's for $s=50$ and $s=100(\mathbf{\square})$. The pore is frictional in a), b) and d).

$N \approx 200, k \sim N^{-\chi}$ and levels off to a constant value $>1$ for longer polymers. At all times, the drag force, $f_{d}$, balances with the constant pore force. $f_{d}$ is exerted on mobile beads, so in the absence of hydrodynamics $f_{d} \sim s_{m}\langle v\rangle$, where $\langle v\rangle$ is the average velocity of the mobile beads. When the whole chain has translocated, $f_{d} \sim N_{m}\langle v\rangle$, where $N_{m}=k N$. With no hydrodynamics, the beads are set in motion from their equilibrium positions, so the distance $d$ of the mobile bead furthest from the pore scales as $d \sim N^{\nu}$. The average translocation time then scales as $\tau \sim\langle d\rangle /\langle v\rangle \sim k N^{1+\nu} \sim N^{1+\nu-\chi}$. For the data in Fig. 2 b), where $f=3$, we obtain $\chi \approx 0.35$ that accords with the measured $\beta=1.26$, see Fig. $1 \mathrm{~b}$ ). With the pore force $f=100$ the $k$ 's for $s_{m}=k s$ are smaller and the measured $s_{m}$ $s$ curves for different $N$ appear more aligned. Asymptotically, $k \rightarrow 1, \forall N$, as $f \rightarrow \infty$, i.e. polymer beads are translocated at the same rate that they are set in motion. Removing the friction from the pore also makes $k$ values smaller and more identical for different $N$ due to translocation becoming faster. Both the increase in the pore force and reduction in the pore friction take the scaling exponent $\beta$ toward $1+\nu$ due to $s_{m}$ and hence the drag force, $f_{d}$, remaining more constant throughout the translocation. Hydrodynamics changes the form of the drag force. $f_{d}$ no more depends strictly linearly on $s_{m}$ for configurations of moving polymer segments, but all beads are set in motion in the beginning of translocation. Yet, the above described mechanism is still clearly underlying the translocation also when hy- drodynamics is included, see Fig. (2]a). For a constant $f$ hydrodynamics reduces $\beta$ due to enhancing collective motion of the polymer towards the pore. Hence, the initial equilibrium positions do not determine $\beta$ like in the absence of hydrodynamics.

In addition to the above described dynamics on the cis side, there is a potential contribution from the crowding of the polymer beads close to the pore on the trans side. This indeed can be seen from the snapshot in Fig. 2 $\mathrm{c}$ ) and the measured radii of gyration, $R_{g}$, of the translocated parts of the polymer on the trans side, which are clearly smaller than the corresponding equilibrium $R_{g}$ even for the smallest force values, see Fig. 2]d). The crowding is more enhanced for longer polymers thus increasing $\beta$, in agreement with our measured translocation times, Figs. (1 b) and c). Asymptotically, for $N \rightarrow \infty$, the average velocity of the polymer beads on the cis side, $\langle v\rangle$, would have to diminish as $s_{m}$ increases to maintain the force balance $f_{d}=f_{p}=$ constant. Eventually $s_{m}$ would be so large that the polymer barely moves, which would bring the translocation to the regime where diffusive motion of the beads shows. For finite polymers this is not the case. Instead, the simulated polymers whose lengths measured in Kuhn lengths are well in the range of polymers used in experiments clearly show that the moving polymer segment does not slow down to velocities where diffusive motion could be seen. Crowding was also reported in the LB simulations in [13]. However, the alignment of segments in the vicinity of the pore, Fig. 2 c), was not observed, but on the contrary polymers were reported to stick to the wall on the cis side.

In conclusion, we have studied forced polymer translocation by a model where hydrodynamics is taken judiciously into account. In our minimal model the pore is non-aqueous, which precludes hydrodynamic coupling of the two chambers separated by the wall, and the pore potential is the only driving force for the translocation. No additional mechanisms for preventing the translocated polymer segments from sliding back to the $c i s$ side were included. Using this model, the smallest pore force at which polymers translocate was estimated to be in the order of $1 \mathrm{pN}$, according with experimental findings [2,3,25]. The used pore force values cover the biologically relevant range and thus characterise well the essential dynamics of forced translocation in biological systems, and DNA experiments. Hydrodynamics was shown to significantly speed up the translocation and diminish variation in the translocation times. The scaling exponent, $\beta$, of the translocation time with respect to polymer length was seen to increase with pore force, $f$. The obtained scaling exponents and their variation with the pore force could be explained by simple force balance at the pore and the observation that the rate, at which the size of the part of the polymer in cis side moving toward the pore grows with respect to the part translocated to the trans side, varies with $f$. A simple estimate was given for the case when hydrodynamics is not included, which agreed with the numerical results. Consequently, a single universal exponent cannot describe translocation for all $f$. The magnitude of pore friction was also seen to change $\beta$. Linear scaling, $\beta=1$, was obtained for large enough friction. $\beta$ was shown to change also due to crowding mechanism on the trans side. As crowding increases with pore force it is an ad- 
ditional mechanism accounting for the increase of $\beta$ with $f$. In summary, by using realistic dynamics where hydrodynamics is included we have shown that experimentally observed forced translocation can be described by a simple force balance. The forced translocation process was also shown to be a highly non-equilibrium process for the experimentally relevant force regime, which explains the discrepancy between theoretical approaches and experiments. We have shown that no universal scaling of the translocation time with the polymer length exists. Experimental verification of this by using different pore potential magnitudes would be very important.

$$
* * *
$$

This work has been supported by the Academy of Finland (Project No. 127766).

\section{REFERENCES}

[1] Alberts B. et al., Molecular Biology of the Cell (Garland Publishing, New York) 1994.

[2] Storm A. J., Storm C., Chen J., Zandbergen H., JoAnny J.-F. and DEKKER C., Nano Lett. , 5 (2005) 1193.

[3] Li J., Gershow M., STEIN D., BRANDin E. and Golovchenko J. A., Nature Materials , 2 (2003) 611.

[4] Meller A., J. Phys.: Condens. Matter, 15 (2003) R581.

[5] Kasianowicz J. J., BRANDin E., BRANTON D. and DeAmer D. W., Proc. Natl. Acad. Sci. U.S.A. , 93 (1996) 13770.

[6] Sung W. and Park P. J., Phys. Rev. Lett. , 77 (1996) 783.

[7] Chuang J., Kantor Y. and Kardar M., Phys. Rev. E, 65 (2001) 011802.

[8] Kantor Y. and Kardar M., Phys. Rev. E, 69 (2004) 021806.

[9] Ali I. and Yeomans J. M., J. of Chem. Phys. , 123 (2005) 234903.

[10] Gauthier M. and Slater G., Eur. Phys. J. E , 25 (2008) 17.

[11] Lehtola V. V., Linna R. P. and Kaski K., Phys. Rev. E, 78 (2008) 061803.

[12] Bernaschi M., Melchionna S., Succi S., Fyta M. and KAXIRAS E., Nanoletters, 8 (2008) 1115.

[13] Fyta, M. E., Melchionna S., Succi S. and KaXiras, Phys. Rev. E, 78 (2008) 036704.

[14] Malevanets A. and Kapral R., J. of Chem. Phys., 110 (1999) 8605.

[15] Ihle T. and Kroll D. M., Phys. Rev. E, 63 (2000) 020201.

[16] Webster M. A. and Yeomans J. M., J. of Chem. Phys. , 122 (2005) 164903.

[17] Linna R. P. and Kaski K., Phys. Rev. Lett. , 100 (2008) 168104.

[18] Ali I., Marenduzzo D. and Yeomans J. M., J. of Chem. Phys. , 121 (2004) 8635.

[19] Panja D., Barkema G. T. and Ball R. C., J. Phys.: Condens. Matter, 19 (2007) 432202.

[20] Muthukumar M., J. of Chem. Phys. , 111 (1999) 10371.

[21] Dubbeldam J. L. A., Milchev A., Rostiashvili V. G. and VILGIS T. A., Europhys. Lett. , 79 (2007) 18002.

[22] Tinland B., Pluen A., Sturm J. and Weill G., Macromol. , 30 (1997) 5763.

[23] Manning G., Q. Rev. Biophys. , 11 (1978) 179.
[24] Sauer-Budge A. F., Nyamwanda J. A., Lubensky D. K. and Branton D., Phys. Rev. Lett. , 90 (2003) 238101.

[25] VAN DER LAAN et al. M., Nature Cell Biol. , 9 (2007) 1152.

[26] Shariff K., Ghosal S. and MatouscheK A., Biophys. J. , 86 (2004) 3647.

[27] Huang S., Ratliff K. S. and Matouschek A., Nature Struct. Biol. , 9 (2002) 301.

[28] Chen L. B., Ann. Rev. Cell Biol. , 4 (1988) 155.

[29] Kikuchi N., Pooley C. M., Ryder J. F. and Yeomans J. M., J. of Chem. Phys., 119 (2003) 6388.

[30] DoI M. and EDWARDS S. F., The Theory of Polymer Dynamics (Oxford science publications, Oxford) 1986.

[31] Fyta M., Kaxiras E., Melchionna S. and Succi S., Comp. Sci. \& Eng. , 2 (2008) 20.

[32] Dubbeldam J. L. A., Milchev A., Rostiashvili V. G. and Vilgis T. A., Phys. Rev. E(R), 76 (2007) 010801. 